\title{
Study on Remote Medical System Application in the Severe Trauma Pre-hospital First Aid
}

\author{
Wu Xiao-qiang, Zhang Chunyou, Wang Li-hua, Li Li \\ ${ }^{1}$ College of Mechanical Engineering, Inner Mongolia University for the Nationalities, \\ Tongliao 028000, China
}

\begin{abstract}
Objective: Study the effects of the application of remote medical system in the severe trauma pre-hospital first aid. Methods: Select Inner Mongolia national university affiliated hospital using remote medical system to rescue 43 emergency patients with new brain stroke patients in June 2014 - September 2015. And 42 emergency patients with severe trauma who are prior to use remote medical system in October 2013 - June 2014 were compared. Results: The arrived at the emergency department time of two groups patients has no statistical significance ( $\mathrm{P}>0.05)$. Remote group has shortened the treatment time than traditional significantly $(\mathrm{P}<0.05)$, and mortality remote also been decreased significantly $(\mathrm{P}<$ 0.05). Conclusions: The application of remote medical system for patients with severe trauma emergency in pre-hospital first aid could make the hospital treatment in advance, shorten the treatment time, combine the hospital and prehospital first aid, and reduce the mortality of severe brain injury.
\end{abstract}

Keywords: Remote Medical System, Pre-hospital First Aid, Information isolated island

\section{Introduction}

There are about 2 million patients with new stroke in China every year, and about 1.5 million people are died of stroke. More than $75 \%$ of survivors have the disability at different degrees, including $40 \%$ of the severe handicapped. Among the people died or disabled of the stroke, many surpass the optimal treatment time window of 6 hours when sent to the hospital for treatment. Therefore, the treatment of patients with stroke must be timely and efficient, and we can try to strive for the survival quality.

The following several points shall be achieved in view of the attack and treatment risk of stroke: Firstly is try to find the potential patients with stroke as early as possible, timely offer the medical advice and control the patient's condition, and prevent the development of the disease; Secondly is to timely get the diagnosis and treatment for the found patients with stroke, the medical agency with low medical level can diagnose and treat the patients with other technical level in the advanced medical agency via sharing the medical resource; Thirdly is to get the online medical experience from other successful recovered patients with stroke for the regional medical agency with relatively backward medical condition, and refer to the treatment of the current patients with stroke; Fourthly is that it is hard to fully recover the patients with stroke after the treatment, the recurrence risk is high with certain sequel, such as the crippled and others, we need to trace the patient's rehabilitation process and timely evaluate the recovery situation and prevent the recurrence of the disease; Fifthly is that 
the treatment of stroke is relatively difficult and the cost is higher, so it needs to use certain technical means to realize the medical resource sharing and reduce the medical cost.

The community health center, 120 emergency center, central hospital, rehabilitation center and other medial agencies may be needed for all patients' medical processes, and a set of mature electronic medical system is equipped with each medical agency currently. For it is the "information isolated island" among the medical systems, it only opens to the internal medical agency, and due to the technology, safety and medical interests relationship and other factors, other medical agency can not interfere into other medical systems. Therefore, the resource among the medical agencies can not be shared in the case of uneven distribution of medical resource, the patient's medical records formed in the medical agencies can not be visited mutually, thus leading to the existence of "information isolated island" among the medical agencies, and causing the huge resource waste.

Starting from the above problem, the remote medical collaborative service topic for the stroke helps the medical activity via the trace of whole medical process for the patients with stroke. For the remote medical treatment is a process across the medical institution and region, so the mutual cooperation among the medical structures or related organizations is the key, it also needs to seamlessly connect and collaborate the corresponding service provided by them, and promote the effective execution of remote medical service for stroke. The goal of the topic is to use the SOA-based idea and system architecture, connect the medical unit, manage the whole process of prevention, first-aid, treatment and diagnosis and rehabilitation, namely collecting, saving and cross-agency sharing of patient's data; notify the patient to check the patient's updated data, timely evaluate and diagnose the patient's data, offer the treatment suggestion and program;

When the patient's disease is in a high risk, the disease can be found early and timely treated; or the patient can be helped timely after calling 120 at the occurrence of the disease; the treatment can be assisted via the remote medical means when treating the patients; continue to monitor the patient's physical condition in the rehabilitation process, timely feedback and treat. The reference and help can be provided for the future disease diagnosis via the patient's previous diagnosis data.

\section{Method and object}

Inner Mongolia national university affiliated hospital was selected to use remote medical system to rescue 43 emergency patients with new brain stroke patients in June 2014 September 2015. And 42 emergency patients with severe trauma who are prior to use remote medical system in October 2013 - June 2014 were compared.

The remote medical collaborative service function is to coordinate the community health center, 120 emergency center, hospital and rehabilitation training center for patient's medical activity. Not every patient with stroke needs to accept all medical activities in the medical agency, it is possible that the collaborative service only occurs between the patient and certain or certain several medical agencies (see Figure 1). 


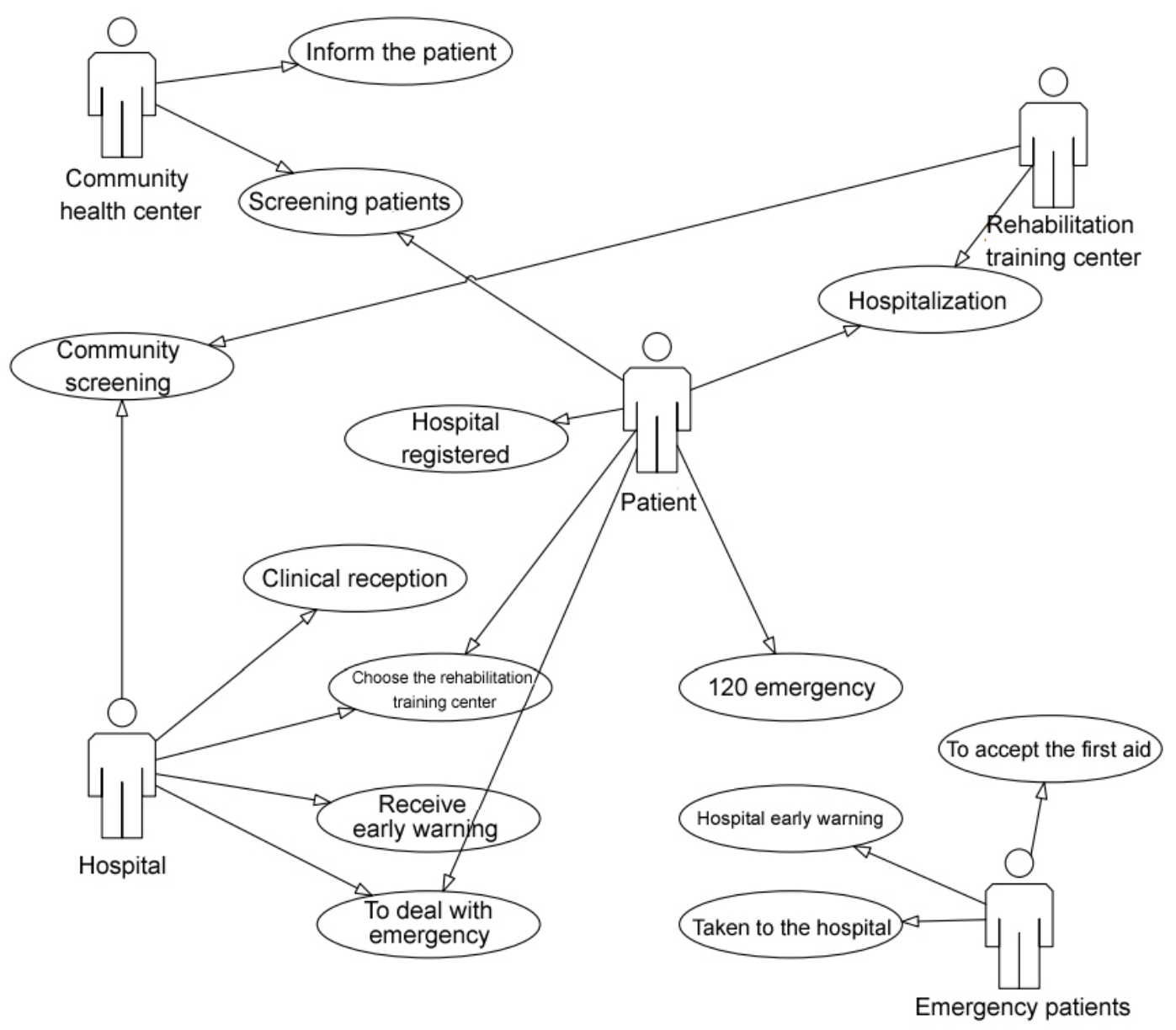

Figure 1 Use case diagram for remote medical collaborative service

The use case diagram for remote medical collaborative service for the patient with stroke is shown in Figure 1. All medical processes and medical activities for the patient with stroke are covered in the figure. After the community health center screens the patient, it will notify the patient to register in the outpatient service for diagnosis if the patient is in a high risk. After the patient receives the notice and registers in the hospital, the hospital will receive. If the patient's disease is slight and the hospitalization is not needed, the hospital will notify the community to screen, and the community health center will arrange the regular screening for the patient after receiving the screening notice. If the patient's disease is severe, the patient needs to be hospitalized for treatment. After the patient completes the treatment in the hospital, if the rehabilitation training is needed, the hospital or patient can actively select the rehabilitation training center, and the rehabilitation training center will handle the checking-in procedure and arrange the rehabilitation training for the patient. If the patient is recovered, the rehabilitation training center will notify the community health center that the patient will return to the screening process of community health center, and the community health center will arrange the regular screening service for the patient. If the patient has the accident, the patient or the contact of patient shall call the emergency phone for the patient, the emergency center will receive the emergency after receiving the emergency phone, select the hospital and send the early-warning for the hospital, and notify the hospital to make the rescue preparation in advance. Meanwhile, the ambulance sent by the emergency center will deliver the patient to the hospital. The hospital receives the patient, handles the emergency registration and arranges the treatment. 


\section{Results and discussion}

The stroke has become the second largest death-causing disease and the largest disability-causing disease in the world. The following several aspects shall be solved to eliminate or reduce the harm of stroke disease for people: preventing in advance is to try to find the potential patient as early as possible; the shortest emergency is to deliver the patient to the hospital for treatment at the optimal emergency time window of 6 hours of stroke; getting the accurate diagnosis as soon as possible; and getting the effective treatment and caring after the diagnosis. However, the "information isolated island" is formed among the medical agencies currently, and it is hard to communicate, therefore, the collaborative medical on the prevention, diagnosis, emergency, treatment and recovery can not be achieved after the patient has the stroke, thus causing the waste of resource, and the difficulty to get the effective health care.

In order to solve the above problems, the topic aims to provide the convenience for the collaborative and communication among the medical agencies as the auxiliary medical system based on remaining the original system. Therefore, the four-aspect work is made for the topic:

(1) Via analyzing all medical activities of patient with stroke, the process model is established for the medical activity of stroke, the whole medical activities are divided into five processes of monitoring and prevention, emergency and delivery, inspection and diagnosis, treatment and caring, recovery and caring. The application of Web Service and WS-BPEL technology based on SOA opens the interface of foreign access in each medical system and connects with the way of BPEL process. The monitoring on the whole medical activities of the patient with stroke is realized, and it can help the doctor to check the patient's medical activity at any time.

(2) The collaborative service is occurred in the edge of medical process and internal HSP system. Via identifying the interaction among the medical processes and making the Web Service way as the resource scheduling of collaborative service among the processes, the collaborative service such as registration and collaborative diagnosis among the medical agencies can be realized.

(3) The reference and prevention support can be provided for the doctor's diagnosis and treatment of the patients via combing the information resource in each medical agency and finding the similar patients and counting the disease.

\section{Conclusions}

To sum up, the SOA architecture is applicable to the information interaction among the medical systems with the type of "information isolated island", which can effectively communicate with the medical system and achieve the collaborative service among the medical agencies. The application of remote medical system for patients with severe trauma emergency in pre-hospital first aid could make the hospital treatment in advance, shorten the treatment time, combine the hospital and prehospital first aid, and reduce the mortality of severe brain injury.

\section{Acknowledgements}

Supported by the Inner Mongolia Autonomous Region Natural Science Foundation (2016MS0550). 


\section{Reference}

[1] Eisenburger P, Safar P. Life supporting first aid training of the public_-review and recommendations[J]. Resuscitation, 1999, 41(1): 3-18.

[2] Haghparast-Bidgoli, Hassan, et al. "Barriers and facilitators to provide effective pre-hospital trauma care for road traffic injury victims in Iran: a grounded theory approach." BMC emergency medicine 10.1 (2010): 20.

[3] Allison, Keith, and Keith Porter. "Consensus on the pre-hospital approach to burns patient management." Accident and emergency nursing 12.1 (2004): 53-57.

[4] Koehler, John J., et al. "Prehospital Index: a scoring system for field triage of trauma victims." Annals of emergency medicine 15.2 (1986): 178-182.

[5] Fatovich, Daniel M., et al. "A comparison of metropolitan vs rural major trauma in Western Australia." Resuscitation 82.7 (2011): 886-890.

[6] Su, Mei-Ju, et al. "Application of tele-ultrasound in emergency medical services." Telemedicine and e-Health 14.8 (2008): 816-824.

[7] Llewellyn, Craig H. "Triage: In austere environments and echeloned medical systems." World journal of surgery 16.5 (1992): 904-909. 